\title{
Novel Structural Hybrids of Pyrrole and Thiazole Moieties: Synthesis and Evaluation of Antibacterial and Antifungal Activities
}

\author{
Mohamed A. Salem, ${ }^{1}$ Samir Y. Abbas,,${ }^{2,}$ Marwa A. M. Sh. El-Sharief, ${ }^{1,3}$ \\ Mohamed H. Helal, ${ }^{4}$ Moustafa A. Gouda, ${ }^{5}$ Mohammed A. Assiri, ${ }^{6}$ \\ and Tarik E. Ali ${ }^{6,7}$ \\ ${ }^{1}$ Department of Chemistry, Faculty of Science and Arts, King Khalid University, Mohail, Assir, Saudi Arabia \\ ${ }^{2}$ Organometallic and Organometalloid Chemistry Department, National Research Centre, Cairo, Egypt \\ ${ }^{3}$ Applied Organic Chemistry Department, National Research Centre, Cairo, Egypt \\ ${ }^{4}$ Department of Chemistry, Faculty of Arts and Science, Northern Border University, Rafha, KSA \\ ${ }^{5}$ Department of Chemistry, Faculty of Science and Arts, Ulla, Taibah University, Medina, Saudi Arabia \\ ${ }^{6}$ Department of Chemistry, Faculty of Science, King Khalid University, Abha, Saudi Arabia \\ ${ }^{7}$ Department of Chemistry, Faculty of Education, Ain Shams University, Cairo, Egypt \\ *Corresponding author: E-mail: samiryoussef98@yahoo.com; sy.abbas@nrc.sci.eg
}

Received: 06-01-2021

\begin{abstract}
One of the best ways to design new biocidal agents is synthesizing hybrid molecules by combining two or more bioactive moieties in a single molecular scaffold. So, new series of pyrroles bearing a thiazole moiety were synthesized using 1-methyl-1 $H$-pyrrole-2-carbaldehyde thiosemicarbazones 1a-c. Cyclization of thiosemicarbazone derivatives $\mathbf{1 a}-\mathbf{c}$ with ethyl chloroacetate, ethyl 2-chloropropanoate, chloroacetone and phenacyl bromide afforded the corresponding thiazolidin-4-ones 2a-c, 5-methylthiazolidin-4-ones 3a-c, 4-methyl-2,3-dihydrothiazoles 4a-c, and 4-phenyl-2,3-dihydrothiazoles $\mathbf{5} \mathbf{a}-\mathbf{c}$, respectively. The antimicrobial activity of the new thiazole derivatives was evaluated.
\end{abstract}

Keywords: Pyrroles; Thiosemicarbazones; Thiazoles; Antibacterial activity; Antifungal activity

\section{Introduction}

One of the most serious future challenges to health care professionals is the emergence of multi-drug resistance pathogenic bacteria that rapidly develop resistance to currently used antibiotics. These medical problems can be reduced by the discovery of novel antibacterial and antifungal agents. ${ }^{1-10}$

Pyrrole is widely known as a biologically active scaffold which possesses a diverse nature of activities. ${ }^{11,12}$ The combination of different pharmacophores in a pyrrole ring system has led to the formation of more active compounds. ${ }^{12}$ The marketed drugs containing a pyrrole ring system are known to have many biological properties such as antipsychotic, $\beta$-adrenergic antagonist, anxiolytic, anticancer (leukemia, lymphoma and myelofibrosis etc.), antibacterial, antifungal, antiprotozoal, antimalarial and many more. ${ }^{13-15}$ Pyrrolomycins and $\mathrm{N}$-alkylated derivatives of pyrrolomycin are natural antibiotics and contain nitropyrrole nucleus which is stable and chemically reactive for antifungal activity. ${ }^{16}$ Verrucarine $\mathrm{E}$ and fenpiclonil are therapeutically useful antibacterial compounds. A naturally occurring pyoluteorin was found to posses antibacterial activity. Sortase A is a transpeptidase responsible for covalently anchoring many surface proteins in Gram-positive peptidoglycan, thus encouraging adhesion and biofilm formation. The inhibition of SrtA is related to the at- 
tenuation of virulence in Staphylococcus aureus and also in other important Gram-positive pathogens, such as Listeria monocytogenes, S. pneumoniae, and S. smutans. ${ }^{17,18}$

Thiazole moiety frequently appears in the structure of many natural products as well as biologically active compounds. Thiazole moiety displayed crucial role in the medicinal chemistry research, where they have therapeutic effects against several diseases. Thiazoles showed the broad variety of biological activities, such as anti-inflammatory, ${ }^{19}$ analgesic, ${ }^{20}$ allergies, ${ }^{21}$ hypertension, ${ }^{22}$ hypnotics, ${ }^{23}$ schizophrenia, ${ }^{24}$ anti-cancer, ${ }^{25}$ antibacterial, antifun$\mathrm{gal}^{1}$ and anti-HIV ${ }^{26}$ activities. Thiazole moiety represented the integral scaffold of the all penicillin derivatives. Penicillins are the first effective antibiotics used to treat microbes that played critical roles in the therapy of the bacterial diseases. ${ }^{27}$ Abafungin is a broad-spectrum antifungal agent. Thiabendazole is a fungicide and parasiticide where it represented a new bacterial DNA gyrase B inhibitors. ${ }^{28}$ Furthermore, most of fused thiazoles are attractive scaffolds for obtaining high potential drug candidates. ${ }^{29,30}$ Fused thiazoles, such as purine and their analogs possess significant biological activities. ${ }^{31}$

The synthesized compounds design was based on three points. First point is the many reports validation of pyrrole as antimicrobial agents. Second point, thiazole moiety has been already reported for its antimicrobial activity, thiazoles have occupied a prominent role in the design of biologically active agents. Thirdly, one of the main strategies for the discovery of new drugs is to modify the structure of a known drug or to unite two or more pharmacophoric moieties that being combined in one molecular scaffold to obtain the synergistic effect or developing newly affordable antibacterial activity having a new mode of action. To assemble novel biologically active compounds with potent antimicrobial effect, it is aimed to synthesize and evaluate the antimicrobial activity of series of 2-substituted pyrroles containing thiazole nucleus separated by a hydrophobic linker.

\section{Results and Discussion}

The starting 1-methyl-1H-pyrrole-2-carbaldehyde thiosemicarbazones 1a-cwere synthesized through the condensation reaction between 1-methyl-1H-pyrrole-2-carbaldehyde and thiosemicarbazide derivatives in ethanol under reflux (Scheme 1). ${ }^{1} \mathrm{H}$ NMR spectrum of $\mathbf{1 b}$ indicated doublet and singlet signals at $\delta 3.01$ and $3.80 \mathrm{ppm}$ for $\mathrm{NH}-\mathrm{CH}_{3}$ and $\mathrm{NCH}_{3}$ protons, two signals at $\delta 7.88$ and 11.14 ppm assignable for two $\mathrm{NH}$ protons, in addition to three multiplet signals at $\delta 6.08,6.51$ and $6.94 \mathrm{ppm}$ for pyrrole protons with singlet signal at $\delta 8.04 \mathrm{ppm}$ corresponding to $\mathrm{CH}=\mathrm{N}$ proton. Also, ${ }^{13} \mathrm{C}$ NMR indicated signals at $\delta 31.3$ $\left(\mathrm{CH}_{3}\right), 36.51\left(\mathrm{CH}_{3}\right), 108.73,114.85,127.34,128.28,136.32$ $(\mathrm{CH}=\mathrm{N})$ and $177.49(\mathrm{C}=\mathrm{S}) \mathrm{ppm}$.

Thiosemicarbazones 1a-c were subjected to cycloalkylation with different halogenated compounds in the hope of obtaining biologically activity thiazoles. Thus, when thiosemicarbazones $1 \mathbf{a}-\mathbf{c}$ were left to react with ethyl chloroacetate in refluxing ethanol containing a catalytic amount of fused sodium acetate resulted in the formation of the corresponding thiazolidines $\mathbf{2} \mathbf{a}-\mathbf{c}$ that were obtained in good yields. Evidence for assigned structures is provided by analytical and spectroscopic data. For example, ${ }^{1} \mathrm{H}$ NMR spectrum of $\mathbf{2 a}$, as an example, exhibited two singlet signals at $\delta 3.86$ and $3.87 \mathrm{ppm}$ assignable to $\mathrm{CH}_{3}$ and $\mathrm{CH}_{2}$ protons. The three protons of pyrrole ring were displayed at $\delta 6.12,6.56$ and $7.01 \mathrm{ppm}$ as multiplet, singlet and singlet signals, respectively. A singlet signal at $\delta$ $8.25 \mathrm{ppm}$ appeared for azomethine proton and a broad signal at $\delta 11.84 \mathrm{ppm}$ due to imino proton. The formation of thiazolidone 2 may be assumed to proceed through initial alkylation for thiosemicarbazones $\mathbf{1}$ to afford the non-isolable intermediate $\mathbf{A}$ followed by intramolecular cyclization with elimination of ethanol (Scheme 2).

Similarly, 5-methyl-thiazolidin-4-one derivatives 3a-c were obtained via cycloalkylation of thiosemicarbazones 1a-c with ethyl 2-chloropropanoate in ethanol in the presence of sodium acetate under reflux condition. ${ }^{1} \mathrm{H}$ NMR spectrum of $\mathbf{3 a}$ showed three diagnostic aliphatic signals for $\mathrm{CH}_{3}-\mathrm{CH}, \mathrm{NCH}_{3}$ and $\mathrm{CH}-\mathrm{CH}_{3}$ protons at $\delta 1.49$ (doublet), 3.87 (singlet) and 4.17 (quartet) ppm, respectively. The protons of pyrrole were displayed at $\delta 6.12,6.55$ and $7.10 \mathrm{ppm}$. Beside the last aromatic protons, ${ }^{1} \mathrm{H}$ NMR spectrum displayed signals for azomethine at $\delta 8.25 \mathrm{ppm}$ together with $\mathrm{NH}$ broad signals at $\delta 11.80 \mathrm{ppm}$. In addition, under the same reaction conditions, cyclocondensation of thiosemicarbazones $\mathbf{1 a}-\mathbf{c}$ with chloroacetone furnished the corresponding 4-methyl-2,3-dihydrothiazole derivatives $\mathbf{4 a}-\mathbf{c} .{ }^{1} \mathrm{H}$ NMR spectrum of $\mathbf{4 a}$ exhibited two characteristic singlet signals for two methyl protons at $\delta$ 2.14 and $3.83 \mathrm{ppm}$. The $\mathrm{H}-5$ of thiazole appaered at $\delta 6.06$

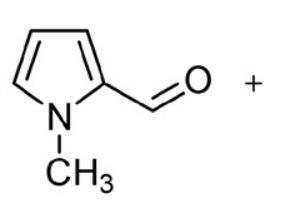<smiles>[R]NC(=S)NN</smiles><smiles>[R][R10]([H])([H])O</smiles><smiles>[R]NC(=S)N/N=C/c1cccn1C</smiles>

Scheme 1: Synthesis of 1-methyl-1H-pyrrole-2-carbaldehyde thiosemicarbazones 1a-c 
<smiles>[R]N=C(S)N/N=C/c1cccn1C</smiles>

1a-c<smiles>[R]NC(=NN=Cc1cccn1C)SCC(=O)OCC</smiles>

A<smiles>[R]N1C(=O)CS/C1=N\N=C\c1cccn1C</smiles>

$$
\begin{aligned}
& \mathbf{1 , 2} \mathbf{a} ; \mathrm{R}=\mathrm{H} \\
& \mathbf{1 , 2} \mathbf{b} ; \mathrm{R}=\mathrm{CH}_{3}, \\
& \mathbf{1 , 2} \mathbf{c} ; \mathrm{R}=\mathrm{Ph}
\end{aligned}
$$

Scheme 2: Synthesis of thiazolidines $2 \mathrm{a}-\mathbf{c}$

ppm. The pyrrole protons were displayed at $\delta 6.28,6.37$ and $6.90 \mathrm{ppm}$, azomethine at $\delta 7.94 \mathrm{ppm}$. A broad signal at $\delta 11.39 \mathrm{ppm}$ appeared for $\mathrm{NH}$ proton. Moreover, cyclocondensation of thiosemicarbazones 1a-c with phenacyl bromide furnished the corresponding 4-phenyl-2,3-dihydrothiazole derivatives $\mathbf{5 a - c . ~}{ }^{1} \mathrm{H}$ NMR spectrum of $\mathbf{5 a}$ showed singlet signal at $\delta 3.87 \mathrm{ppm}$ assignable to $\mathrm{NCH}_{3}$. The protons of phenyl group were displayed at $\delta 6.08,7.40$ and $7.85 \mathrm{ppm}$ as triplet (one proton), triplet (two protons), and doublet (two protons), respectively. The protons of pyrrole were displayed at $\delta 6.93,7.25$ and $7.30 \mathrm{ppm}$. Signals at 6.41 and $7.98 \mathrm{ppm}$ were characteristics for $\mathrm{CH}$-thiazole and $\mathrm{CH}=\mathrm{N}$, respectively and a broad signal at $\delta 11.77$ ppm due to $\mathrm{NH}$ proton (Scheme 3).

The newly synthesized compounds were screened for their expected antifungal and antibacterial activities.
Three different microbial groups were used. Group 1: Gram-positive bacteria: Bacillus subtilis (RCMB 015 (1) NRRL B-543) and Staphylococcus aureus (ATCC 25923). Group 2: Gram-negative bacteria: Proteus vulgaris (RCMB 004 (1) ATCC 13315) and Escherichia coli (ATCC 25922). Group 3: Fungi: Candida albicans (RCMB 005003 (1) ATCC 10231) and Aspergillus fumigatus (RCMB 002008). For the screening of antibacterial activity, diffusion agar technique ${ }^{1}$ was applied at $10 \mathrm{mg} / \mathrm{mL}$ concentration, well diameter $6.0 \mathrm{~mm}(100 \mu \mathrm{L}$ was tested). For comparison, Gentamycin and Ketoconazole were used as antibacterial and antifungal agents, respectively. The inhibition zone diameters are depicted in Table 1. Regarding the antimicrobial activity of thiazole derivatives, they displayed good effects towards $P$. vulgaris only. Few compounds displayed weak effects towards some of the tested organisms. The

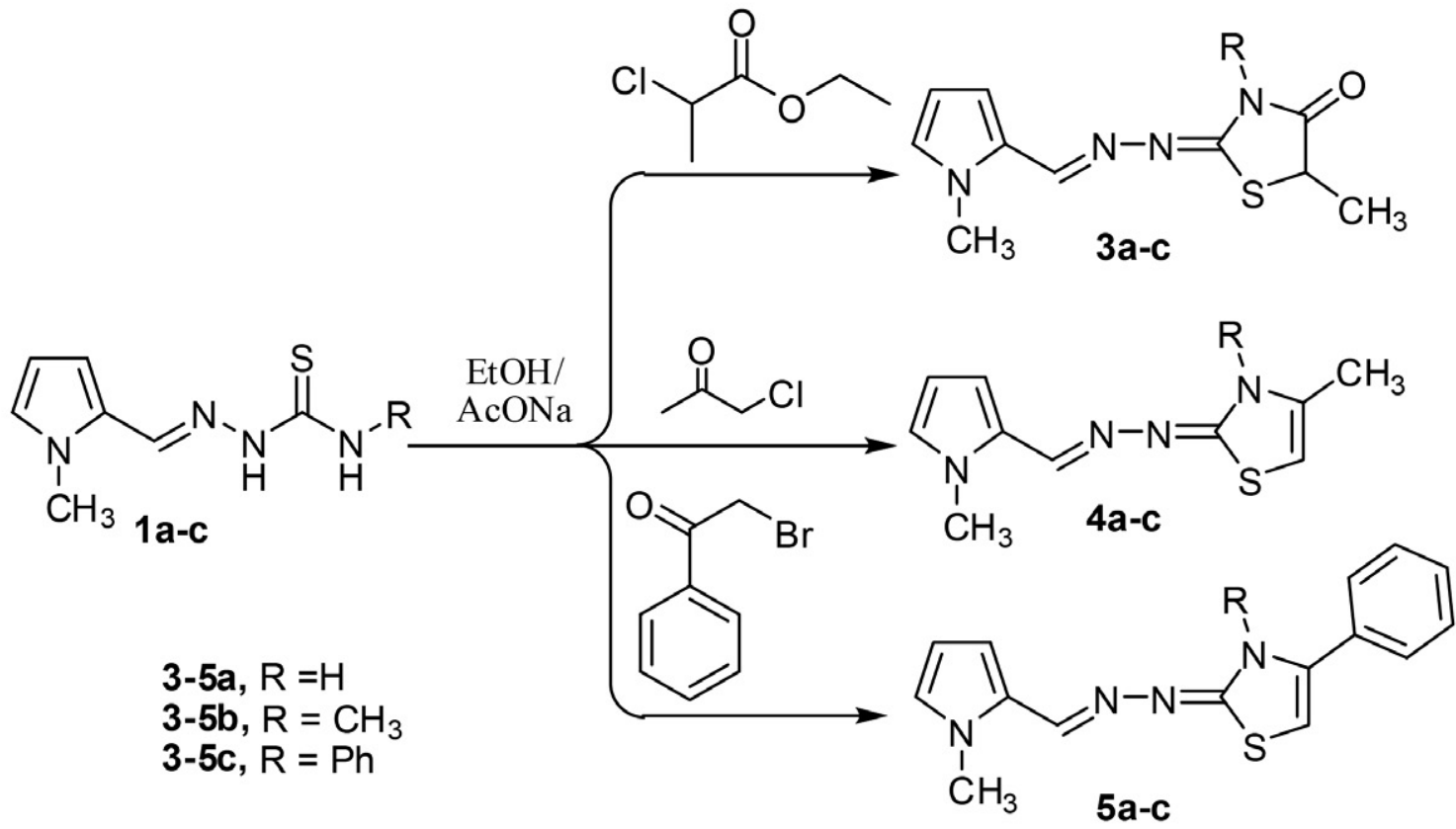

Scheme 3: Synthesis of thiazole derivatives $\mathbf{3 a}-\mathbf{c}, \mathbf{4 a - c}$ and $\mathbf{5 a}-\mathbf{c}$. 
Table 1: The mean results of inhibition zone in mm produced on a range of pathogenic microorganisms

\begin{tabular}{lcccccc}
\hline Compd. No. & \multicolumn{2}{c}{ Gram positive bacteria } & \multicolumn{2}{c}{ Gram negative bacteria } & \multicolumn{2}{c}{ Fungi } \\
& B. subtilis & S. aureus & P. vulgaris & E. coli & C. albicans A. fumigatus \\
\hline $\mathbf{2 a}$ & $\mathrm{NA}$ & $\mathrm{NA}$ & 21 & 10 & $\mathrm{NA}$ & $\mathrm{NA}$ \\
$\mathbf{2 b}$ & $\mathrm{NA}$ & $\mathrm{NA}$ & 23 & 9 & $\mathrm{NA}$ & $\mathrm{NA}$ \\
$\mathbf{2 c}$ & $\mathrm{NA}$ & $\mathrm{NA}$ & 10 & $\mathrm{NA}$ & $\mathrm{NA}$ & $\mathrm{NA}$ \\
$\mathbf{3 a}$ & $\mathrm{NA}$ & $\mathrm{NA}$ & 16 & $\mathrm{NA}$ & $\mathrm{NA}$ & $\mathrm{NA}$ \\
$\mathbf{3 b}$ & 9 & $\mathrm{NA}$ & 15 & 10 & 8 & $\mathrm{NA}$ \\
$\mathbf{3 c}$ & 8 & 8 & 16 & 9 & $\mathrm{NA}$ & 9 \\
$\mathbf{4 a}$ & 12 & $\mathrm{NA}$ & 13 & $\mathrm{NA}$ & 9 & 8 \\
$\mathbf{4 b}$ & $\mathrm{NA}$ & $\mathrm{NA}$ & 24 & 12 & $\mathrm{NA}$ & $\mathrm{NA}$ \\
$\mathbf{4 c}$ & 8 & 9 & 18 & 10 & 8 & 8 \\
$\mathbf{5 a}$ & 9 & $\mathrm{NA}$ & 15 & 13 & $\mathrm{NA}$ & $\mathrm{NA}$ \\
$\mathbf{5 b}$ & $\mathrm{NA}$ & $\mathrm{NA}$ & 18 & 8 & 9 & NA \\
$\mathbf{5 c}$ & 9 & $\mathrm{NA}$ & 17 & 9 & - & 8 \\
Gentamycin & 26 & 24 & 25 & 30 & 20 & 17 \\
Ketoconazole & - & - & - & - & & - \\
\hline
\end{tabular}

major compounds displayed no effects towards most of the tested organisms.

\section{Conclusion}

New series of pyrroles bearing a thiazole moiety were synthesized through cyclization of 1-methyl- $1 H$-pyrrole-2-carbaldehyde thiosemicarbazone derivatives with ethyl chloroacetate, ethyl 2-chloropropanoate, chloroacetone and phenacyl bromide. The thiazole derivatives displayed good effects towards $P$. vulgaris only. Few compounds displayed weak effects towards some of the tested organisms. The major compounds displayed no effects towards most of the tested organisms.

\section{1. Experimental Section}

Nuclear magnetic resonance spectra were carried out in deuterated dimethylsulfoxide (DMSO- $d_{6}$ ) by using Bruker spectrometers $\left({ }^{1} \mathrm{H}\right.$ NMR $400 \mathrm{MHz} ;{ }^{13} \mathrm{C}$ NMR 101 $\mathrm{MHz}$ ) with chemical shift in $\delta$ from internal TMS. Mass spectra were recorded on GC/MS Finnigan SSQ 7000 spectrophotometer and GC Ms-QP 1000 EX mass spectrometer at $70 \mathrm{eV}$. Elemental analyses were carried out on a EuroVector instrument $\mathrm{C}, \mathrm{H}, \mathrm{N}$ analyzer EA3000 Series.

Synthesis of $\mathrm{N}$-Substituted-2-((1-methyl- $1 \mathrm{H}$-pyrrol-2yl)methylene)hydrazinecarbothioamides $1 \mathrm{a}-\mathrm{c}^{32}$

The mixture of $0.01 \mathrm{~mol}$ of 1 -methyl- $1 \mathrm{H}$-pyrrole2-carbaldehyde and $0.01 \mathrm{~mol}$ of the selected thiosemicarbazides (thiosemicarbazide, $\mathrm{N}$-methylthiosemicarbazide, $\mathrm{N}$-phenylthiosemicarbazide) was heated in $50 \mathrm{~mL}$ ethanol under reflux for $0.5 \mathrm{~h}$., left to cool, the resultant solid product was collected by filtration. The solid products were crystallized from ethanol.
$\mathrm{N}$-Methyl-2-((1-methyl-1H-pyrrol-2-yl)methylene)hydrazinecarbothioamide (1b). Yield $1.76 \mathrm{~g}$ (90\%); m.p. 180-182 ${ }^{\circ} \mathrm{C} ;{ }^{1} \mathrm{H}$ NMR $\delta 3.01\left(\mathrm{~d}, 3 \mathrm{H}, J=4.6 \mathrm{~Hz}, \mathrm{NHCH}_{3}\right)$, $3.80\left(\mathrm{~s}, 3 \mathrm{H}, \mathrm{NCH}_{3}\right), 6.08(\mathrm{~m}, 1 \mathrm{H}$, pyrrole- $\mathrm{H}), 6.51(\mathrm{~m}, 1 \mathrm{H}$, pyrrole-H), $6.94(\mathrm{~m}, 1 \mathrm{H}$, pyrrole-H), $7.88(\mathrm{br}, 1 \mathrm{H}, \mathrm{NH})$, $8.04(\mathrm{~s}, 1 \mathrm{H}, \mathrm{CH}=\mathrm{N}), 11.14(\mathrm{~s}, 1 \mathrm{H}, \mathrm{NH}) ;{ }^{13} \mathrm{C}$ NMR: $\delta 31.3$ $\left(\mathrm{CH}_{3}\right), 36.51\left(\mathrm{CH}_{3}\right), 108.73,114.85,127.34,128.28,136.32$ $(\mathrm{CH}=\mathrm{N}), 177.49(\mathrm{C}=\mathrm{S}) ; \mathrm{MS} m / z(\%): 196\left(\mathrm{M}^{+} ; 67.4\right)$. Anal. Calcd. for $\mathrm{C}_{8} \mathrm{H}_{12} \mathrm{~N}_{4} \mathrm{~S}$ (196.27): C, 48.96; H, 6.16; N, 28.55 . Found: C, 48.87; H, 6.14; N, 28.47\%.

$\mathrm{N}$-Methyl-2-((1-methyl-1H-pyrrol-2-yl)methylene)hydrazinecarbothioamide (1c). Yield $2.45 \mathrm{~g}$ (95\%); m.p. 140-142 ${ }^{\circ} \mathrm{C} ;{ }^{1} \mathrm{H}$ NMR: $\delta 3.86\left(\mathrm{~s}, 3 \mathrm{H}, \mathrm{NCH}_{3}\right), 6.12(\mathrm{~m}$, $1 \mathrm{H}$, pyrrole- $\mathrm{H}), 6.64(\mathrm{~m}, 1 \mathrm{H}$, pyrrole- $\mathrm{H}), 6.99(\mathrm{~m}, 1 \mathrm{H}$, pyrrole-H), 7.18 (t, $1 \mathrm{H}, J=7.4 \mathrm{~Hz}, \mathrm{Ph}-\mathrm{H}), 7.35(\mathrm{t}, 2 \mathrm{H}, J$ $=7.8 \mathrm{~Hz}, \mathrm{Ph}-\mathrm{H}), 7.64(\mathrm{~d}, 2 \mathrm{H}, J=7.7 \mathrm{~Hz}, \mathrm{Ph}-\mathrm{H}), 8.15(\mathrm{~s}$, $1 \mathrm{H}, \mathrm{CH}=\mathrm{N}), 9.55(\mathrm{~s}, 1 \mathrm{H}, \mathrm{NH}), 11.55(\mathrm{~s}, 1 \mathrm{H}, \mathrm{NH}) ; \mathrm{MS} \mathrm{m} / z$ (\%): $258\left(\mathrm{M}^{+} ; 46.0\right)$. Anal. Calcd. for $\mathrm{C}_{13} \mathrm{H}_{14} \mathrm{~N} 4 \mathrm{~S}(258.34)$ : C, 60.44; H, 5.46; N, 21.69. Found: C, 60.38; H, 5.45; N, $21.73 \%$.

\section{Synthesis of Thiazolidin-4-one Derivatives 2a-c}

The mixture of $0.01 \mathrm{~mol}$ of the thiosemicarbazide derivatives $\mathbf{1 a}-\mathbf{c}, 0.01 \mathrm{~mol}$ of ethyl chloroacetate and 0.02 mol of fused sodium acetate was dissolved $50 \mathrm{~mL}$ ethanol. The solution was heated under reflux for 3 hours, then left to cool. The obtained products were collected by filtration. The solid products were recrystallized from ethanol.

2-(((1-Methyl-1H-pyrrol-2-yl)methylene)hydrazono)thiazolidin-4-one (2a). Yield $1.89 \mathrm{~g}$ (85\%); m.p. $248-250{ }^{\circ} \mathrm{C}$; ${ }^{1} \mathrm{H}$ NMR: $\delta 3.86,3.87\left(2 \mathrm{~s}, 5 \mathrm{H}, \mathrm{CH}_{2}\right.$ and $\left.\mathrm{CH}_{3}\right), 6.12(\mathrm{~m}, 1 \mathrm{H}$, pyrrole-H), $6.56(\mathrm{~s}, 1 \mathrm{H}$, pyrrole-H), $7.01(\mathrm{~s}, 1 \mathrm{H}$, pyrrole- $\mathrm{H})$, 8.25 (s, $1 \mathrm{H}, \mathrm{CH}=\mathrm{N}), 11.84$ (s, $1 \mathrm{H}, \mathrm{NH}) ; \mathrm{MS} m / z$ (\%): 222 $\left(\mathrm{M}^{+}\right.$; 33.8). Anal. Calcd. for $\mathrm{C}_{9} \mathrm{H}_{10} \mathrm{~N}_{4} \mathrm{OS}$ (222.27): $\mathrm{C}$, 48.63; H, 4.53; N, 25.21. Found: C, 48.57; H, 4.55; N, 25.17\%. 
3-Methyl-2-(((1-methyl-1H-pyrrol-2-yl)methylene)hydrazono)thiazolidin-4-one (2b). Yield $1.88 \mathrm{~g}(80 \%)$; m.p. 183-184 ${ }^{\circ} \mathrm{C} ;{ }^{1} \mathrm{H}$ NMR: $\delta 3.16$ (s, $\left.3 \mathrm{H}, \mathrm{CH}_{3}\right), 3.88(\mathrm{~s}, 3 \mathrm{H}$, $\left.\mathrm{CH}_{3}\right), 3.92\left(\mathrm{~s}, 2 \mathrm{H}, \mathrm{CH}_{2}\right.$-thiazole), $6.13(\mathrm{~s}, 1 \mathrm{H}$, pyrrole- $\mathrm{H})$, $6.59(\mathrm{~s}, 1 \mathrm{H}$, pyrrole- $\mathrm{H}), 7.03(\mathrm{~s}, 1 \mathrm{H}$, pyrrole- $\mathrm{H}), 8.35(\mathrm{~s}$, $1 \mathrm{H}, \mathrm{CH}=\mathrm{N}) ;{ }^{13} \mathrm{C}$ NMR: $\delta 29.77\left(\mathrm{CH}_{3}\right), 32.54\left(\mathrm{CH}_{3}\right), 37.02$ $\left(\mathrm{CH}_{2}\right), 109.00,117.49,127.55,129.74,150.00(\mathrm{CH}=\mathrm{N})$, $162.69(\mathrm{CH}=\mathrm{N}), 172.61(\mathrm{C}=\mathrm{O}) ; \mathrm{MS} m / z(\%): 236\left(\mathrm{M}^{+}\right.$; 62.2). Anal. Calcd. for $\mathrm{C}_{10} \mathrm{H}_{12} \mathrm{~N}_{4} \mathrm{OS}$ (236.29): $\mathrm{C}, 50.83 ; \mathrm{H}$, 5.12; N, 23.71. Found: C, 50.86; H, 5.10; N, 23.67\%.

2-(((1-Methyl-1H-pyrrol-2-yl)methylene)hydrazono)-3-phenylthiazolidin-4-one (2c). Yield $2.53 \mathrm{~g}$ (85\%); m.p. $256-258{ }^{\circ} \mathrm{C} ;{ }^{1} \mathrm{H}$ NMR: $\delta 3.88\left(\mathrm{~s}, 3 \mathrm{H}, \mathrm{CH}_{3}\right), 4.07$ (s, $2 \mathrm{H}, \mathrm{CH}_{2}$-thiazole), $6.10(\mathrm{~m}, 1 \mathrm{H}$, pyrrole- $\mathrm{H}), 6.53(\mathrm{~m}, 1 \mathrm{H}$, pyrrole- $\mathrm{H}), 7.02(\mathrm{~s}, 1 \mathrm{H}$, pyrrole- $\mathrm{H}), 7.38(\mathrm{~d}, 2 \mathrm{H}, J=7.2$ $\mathrm{Hz}, \mathrm{Ph}-\mathrm{H}), 7.46$ (d, 1H, J = 7.3 Hz, Ph-H), 7.52 (t, 2H, $J=$ $7.4 \mathrm{~Hz}, \mathrm{Ph}-\mathrm{H}), 8.15(\mathrm{~s}, 1 \mathrm{H}, \mathrm{CH}=\mathrm{N})$; MS $m / z(\%): 298\left(\mathrm{M}^{+}\right.$; 22.3). Anal. Calcd. for $\mathrm{C}_{15} \mathrm{H}_{14} \mathrm{~N}_{4} \mathrm{OS}$ (298.36): C, 60.38; H, 4.73; N, 18.78. Found: C, 60.43; H, 4.75; N, 18.82\%.

\section{Synthesis of 5-Methyl-thiazolidin-4-one Derivatives 3a-c}

The mixture of $0.01 \mathrm{~mol}$ of the thiosemicarbazide derivatives 1a-c, $0.01 \mathrm{~mol}$ of ethyl 2-chloropropanoate and $0.02 \mathrm{~mol}$ of fused sodium acetate was dissolved $50 \mathrm{~mL}$ ethanol. The solution was heated under reflux for 5 hours. After cooling, precipitated solid were obtained which were collected by filtration and recrystallized from ethanol.

5-Methyl-2-(((1-methyl-1H-pyrrol-2-yl)methylene)hydrazono)thiazolidin-4-one (3a). Yield $1.65 \mathrm{~g}(70 \%)$; m.p. 210-212 ${ }^{\circ} \mathrm{C}$; ${ }^{1} \mathrm{H}$ NMR: $\delta 1.49\left(\mathrm{~d}, 3 \mathrm{H}, J=7.2 \mathrm{~Hz}, \mathrm{CH}_{3}\right)$, $3.87\left(\mathrm{~s}, 3 \mathrm{H}, \mathrm{NCH}_{3}\right), 4.17(\mathrm{q}, 1 \mathrm{H}, J=7.2 \mathrm{~Hz}$, thiazole- $\mathrm{H})$, $6.12(\mathrm{~m}, 1 \mathrm{H}$, pyrrole- $\mathrm{H}), 6.55(\mathrm{~m}, 1 \mathrm{H}$, pyrrole- $\mathrm{H}), 7.10(\mathrm{~s}$, $1 \mathrm{H}$, pyrrole-H), $8.25(\mathrm{~s}, 1 \mathrm{H}, \mathrm{CH}=\mathrm{N}), 11.80(\mathrm{br}, 1 \mathrm{H}, \mathrm{NH})$; MS $m / z(\%): 236\left(\mathrm{M}^{+} ; 49.0\right)$. Anal. Calcd. for $\mathrm{C}_{10} \mathrm{H}_{12} \mathrm{~N}_{4} \mathrm{OS}$ (236.29): C, 50.83; H, 5.12; N, 23.71. Found: : C, 50.86; H, $5.11 ; \mathrm{N}, 23.67 \%$.

3,5-Dimethyl-2-(((1-methyl-1H-pyrrol-2-yl)methylene)hydrazono)thiazolidin-4-one (3b). Yield $1.68 \mathrm{~g}$ (67\%); ${ }^{1} \mathrm{H}$ NMR: $\delta 1.50$ (d, $3 \mathrm{H}, J=7.2 \mathrm{~Hz}, \mathrm{CH}_{3}$ ), 3.27 (s, $\left.3 \mathrm{H}, \mathrm{CH}_{3}\right), 3.89\left(\mathrm{~s}, 3 \mathrm{H}, \mathrm{CH}_{3}\right), 4.20(\mathrm{~m}, 1 \mathrm{H}$, thiazole- $\mathrm{H}), 6.14$ $(\mathrm{m}, 1 \mathrm{H}$, pyrrole- $\mathrm{H}), 6.55(\mathrm{~m}, 1 \mathrm{H}$, pyrrole- $\mathrm{H}), 7.13(\mathrm{~s}, 1 \mathrm{H}$, pyrrole-H), $8.25(\mathrm{~s}, 1 \mathrm{H}, \mathrm{CH}=\mathrm{N})$; MS $\mathrm{m} / \mathrm{z}(\%): 250\left(\mathrm{M}^{+}\right.$; 18.7). Anal. Calcd. for $\mathrm{C}_{11} \mathrm{H}_{14} \mathrm{~N}_{4} \mathrm{OS}$ (250.32): C, 52.78; $\mathrm{H}$, 5.64; N, 22.38. Found: C, 52.73; H, 5.61; N, 22.42\%.

5-Methyl-2-(((1-methyl-1H-pyrrol-2-yl)methylene)hydrazono)-3-phenylthiazolidin-4-one (3c). Yield $2.34 \mathrm{~g}$ (75\%); ${ }^{1} \mathrm{H}$ NMR: $\delta 1.51\left(\mathrm{~d}, 3 \mathrm{H}, J=7.2 \mathrm{~Hz}, \mathrm{CH}_{3}\right), 3.86$ (s, $3 \mathrm{H}$, $\left.\mathrm{NCH}_{3}\right), 4.22(\mathrm{~m}, 1 \mathrm{H}$, thiazole-H), $6.10-7.10(\mathrm{~m}, 8 \mathrm{H}, \mathrm{Ph}-\mathrm{H}$ and pyrrole-H), $8.32(\mathrm{~s}, 1 \mathrm{H}, \mathrm{CH}=\mathrm{N}) ; \mathrm{MS} m / z(\%): 312\left(\mathrm{M}^{+}\right.$; 27.0). Anal. Calcd. for $\mathrm{C}_{16} \mathrm{H}_{16} \mathrm{~N}_{4} \mathrm{OS}$ (312.39): C, 61.52; $\mathrm{H}$, 5.16; N, 17.93. Found: C, 61.48; H, 5.14; N, 17.96\%.
Synthesis of 4-Methyl-2,3-dihydrothiazole Derivatives $4 a-c$

The mixture of $0.01 \mathrm{~mol}$ of the thiosemicarbazide derivatives $1 \mathbf{a}-\mathbf{c}, 0.01 \mathrm{~mol}$ of chloroacetone and $0.02 \mathrm{~mol}$ of fused sodium acetate was dissolved $50 \mathrm{~mL}$ ethanol. The solution was heated under reflux for $6 \mathrm{~h}$ and left to cool. The obtained product was collected by filtration. The solid products were recrystallized from ethanol.

4-Methyl-2-(((1-methyl-1H-pyrrol-2-yl)methylene)hydrazono)-2,3-dihydrothiazole (4a). Yield $1.65 \mathrm{~g}$ (75\%); m.p. $177-179{ }^{\circ} \mathrm{C} ;{ }^{1} \mathrm{H}$ NMR: $\delta 2.14\left(\mathrm{~s}, 3 \mathrm{H}, \mathrm{CH}_{3}\right.$ ), 3.83 (s, $\left.3 \mathrm{H}, \mathrm{NCH}_{3}\right), 6.06(\mathrm{~m}, 1 \mathrm{H}$, pyrrole- $\mathrm{H}), 6.28(\mathrm{~s}, 1 \mathrm{H}$, thiazole- $\mathrm{H}), 6.37(\mathrm{~m}, 1 \mathrm{H}$, pyrrole- $\mathrm{H}), 6.90(\mathrm{~s}, 1 \mathrm{H}$, pyrrole- $\mathrm{H})$, $7.94(\mathrm{~s}, 1 \mathrm{H}, \mathrm{CH}=\mathrm{N}), 11.39$ (s, $1 \mathrm{H}, \mathrm{NH}) ; \mathrm{MS} m / z(\%): 220$ $\left(\mathrm{M}^{+}\right.$; 46.6). Anal. Calcd. for $\mathrm{C}_{10} \mathrm{H}_{12} \mathrm{~N}_{4} \mathrm{~S}$ (220.29): C, 54.52; H, 5.49; N, 25.43. Found: C, 54.48; H, 5.50; N, 25.38\%.

3,4-Dimethyl-2-(((1-methyl-1H-pyrrol-2-yl)methylene)hydrazono)-2,3-dihydro-thiazole (4b). Yield 1.64 g (70\%); m.p. ${ }^{196-198}{ }^{\circ} \mathrm{C} ;{ }^{1} \mathrm{H}$ NMR: $\delta 2.12\left(\mathrm{~s}, 3 \mathrm{H}, \mathrm{CH}_{3}\right)$, $3.31\left(\mathrm{~s}, 3 \mathrm{H}, \mathrm{NCH}_{3}\right), 3.86\left(\mathrm{~s}, 3 \mathrm{H}, \mathrm{NCH}_{3}\right), 5.95(\mathrm{~m}, 1 \mathrm{H}$, Ar-H), $6.06(\mathrm{~m}, 1 \mathrm{H}, \mathrm{Ar}-\mathrm{H}), 6.39(\mathrm{~m}, 1 \mathrm{H}$, thiazole-H), 6.89 (s, $1 \mathrm{H}, \mathrm{Ar}-\mathrm{H}), 8.17(\mathrm{~s}, 1 \mathrm{H}, \mathrm{CH}=\mathrm{N}) ;{ }^{13} \mathrm{C}$ NMR: $\delta 14.21\left(\mathrm{CH}_{3}\right)$, $31.48\left(\mathrm{CH}_{3}\right), 36.86\left(\mathrm{CH}_{3}\right), 95.69,108.40,114.34,127.78$, $128.77,136.52,143.22,168.37(\mathrm{CH}=\mathrm{N}) ; \mathrm{MS} m / z(\%): 234$ $\left(\mathrm{M}^{+} ; 45.7\right)$. Anal. Calcd. for $\mathrm{C}_{11} \mathrm{H}_{14} \mathrm{~N}_{4} \mathrm{~S}(234.32)$ : $\mathrm{C}, 56.38 ; \mathrm{H}$, $6.02 ; \mathrm{N}, 23.91 ; \mathrm{N}, 17.34$. Found: C, 56.34; H, 6.04; N, 23.87\%.

4-Methyl-2-(((1-methyl-1H-pyrrol-2-yl)methylene)hydrazono)-3-phenyl-2,3-dihydrothiazole (4c). Yield 2.22 $\mathrm{g}(75 \%) ;{ }^{1} \mathrm{H}$ NMR: $\delta 2.15$ (s, 3H, $\left.\mathrm{CH}_{3}\right), 3.85\left(\mathrm{~s}, 3 \mathrm{H}, \mathrm{NCH}_{3}\right.$ ), $5.95(\mathrm{~m}, 1 \mathrm{H}, \mathrm{Ar}-\mathrm{H}), 6.10-6.90(\mathrm{~m}, 8 \mathrm{H}$, pyrrole- $\mathrm{H}$ and $\mathrm{Ph}-$ $\mathrm{H}), 8.26(\mathrm{~s}, 1 \mathrm{H}, \mathrm{CH}=\mathrm{N})$; MS $m / z(\%): 296\left(\mathrm{M}^{+} ; 24.8\right)$. Anal. Calcd. for $\mathrm{C}_{16} \mathrm{H}_{16} \mathrm{~N}_{4} \mathrm{~S}$ (296.39): C, 64.84; H, 5.44; N, 18.90 . Found: C, 64.86; H, 5.42; N, 18.86\%.

\section{Synthesis of 4-Phenyl-2,3-dihydrothiazole Derivatives $5 \mathbf{a}-\mathbf{c}$ \\ The mixture of $0.01 \mathrm{~mol}$ of the thiosemicarbazide derivatives $1 \mathbf{a}-\mathbf{c}, 0.01 \mathrm{~mol}$ of phenacyl bromide and 0.02 mol of fused sodium acetate was dissolved $50 \mathrm{~mL}$ ethanol. The solution was heated under reflux for $6 \mathrm{~h}$ and left to cool. The obtained product was collected by filtration. The solid products were recrystallized from ethanol.}

2-(((1-Methyl-1H-pyrrol-2-yl)methylene)hydrazono)-4-phenyl-2,3-dihydrothiazole (5a). ${ }^{[33]}$ Yield $2.40 \mathrm{~g}$ (85\%); m.p. $180-182{ }^{\circ} \mathrm{C}$; ${ }^{1} \mathrm{H}$ NMR: $\delta 3.87$ (s, $3 \mathrm{H}, \mathrm{CH}_{3}$ ), $6.08(\mathrm{~m}, 1 \mathrm{H}$, pyrrole- $\mathrm{H}), 6.41(\mathrm{~m}, 1 \mathrm{H}$, pyrrole- $\mathrm{H}), 6.93(\mathrm{~s}$, $1 \mathrm{H}$, pyrrole- $\mathrm{H}), 7.25(\mathrm{~s}, 1 \mathrm{H}$, thiazole- $\mathrm{H}), 7.30(\mathrm{~d}, 1 \mathrm{H}, J=$ $7.3 \mathrm{~Hz}, \mathrm{Ph}-\mathrm{H}), 7.40$ (t, $2 \mathrm{H}, J=7.6 \mathrm{~Hz}, \mathrm{Ph}-\mathrm{H}), 7.85$ (d, 2H, $=7.4 \mathrm{~Hz}, \mathrm{Ph}-\mathrm{H}), 7.98(\mathrm{~s}, 1 \mathrm{H}, \mathrm{CH}=\mathrm{N}) ; 11.77(\mathrm{br}, 1 \mathrm{H}, \mathrm{NH})$; MS m/z (\%): $282\left(\mathrm{M}^{+} ; 43.1\right)$. Anal. Calcd. for $\mathrm{C}_{15} \mathrm{H}_{14} \mathrm{~N}_{4} \mathrm{~S}$ (282.36): C, 63.80; H, 5.00; N, 19.84. Found: C, 63.77; H, $4.98 ; \mathrm{N}, 19.78 \%$. 
3-Methyl-2-(((1-methyl-1H-pyrrol-2-yl)methylene)hydrazono)-4-phenyl-2,3-dihydrothiazole (5b). Yield 2.37 g (80\%); m.p. $127-128^{\circ} \mathrm{C} ;{ }^{1} \mathrm{H}$ NMR: $\delta 3.27\left(\mathrm{~s}, 3 \mathrm{H}, \mathrm{CH}_{3}\right)$, $3.89\left(\mathrm{~s}, 3 \mathrm{H}, \mathrm{CH}_{3}\right), 6.06(\mathrm{~m}, 1 \mathrm{H}$, pyrrole- $\mathrm{H}), 6.32(\mathrm{~s}, 1 \mathrm{H}$, thiazole- $\mathrm{H}), 6.43$ (m, 1H,pyrrole- $\mathrm{H}), 6.93$ (s, $1 \mathrm{H}$, pyrrole- $\mathrm{H})$, 7.50 (m, 5H, Ar-H), 8.23 (s, 1H, CH=N); MS m/z (\%): 296 $\left(\mathrm{M}^{+} ; 23.2\right)$. Anal. Calcd. for $\mathrm{C}_{16} \mathrm{H}_{16} \mathrm{~N}_{4} \mathrm{~S}$ (296.39): C, 64.84; H, 5.44; N, 18.90. Found: C, 64.79; H, 5.46; N, 18.87\%.

2-(((1-Methyl-1H-pyrrol-2-yl)methylene)hydrazono)-3,4-diphenyl-2,3-dihydro-thiazole (5c). Yield $3.04 \mathrm{~g}$ (85\%); ${ }^{1} \mathrm{H}$ NMR: $\delta 3.92\left(\mathrm{~s}, 3 \mathrm{H}, \mathrm{CH}_{3}\right), 6.06(\mathrm{~m}, 14 \mathrm{H}$, thiazole- $\mathrm{H}$, pyrrole- $\mathrm{H}, 2 \mathrm{Ph}-\mathrm{H}), 8.34(\mathrm{~s}, 1 \mathrm{H}, \mathrm{CH}=\mathrm{N}) ; \mathrm{MS} \mathrm{m} / \mathrm{z}$ (\%): $358\left(\mathrm{M}^{+} ; 49.0\right)$. Anal. Calcd. for $\mathrm{C}_{21} \mathrm{H}_{18} \mathrm{~N}_{4} \mathrm{~S}$ (358.46): C, 70.36; H, 5.06; N, 15.63. Found: C, 70.41; H, 5.08; N, $15.57 \%$.

\section{Acknowledgment}

The authors extend their appreciation to the Deanship of Scientific Research at King Khalid University for funding this work through General Research Project under grant number (RGP. 2/88/42).

\section{References}

1 S. I. Eissa, A. M. Farrag, S. Y. Abbas, M. F. El Shehry, A. Ragab, E. A. Fayed, Y. A. Ammar, Bioorg. Chem., 2021, 110, 104803 DOI:10.1016/j.bioorg.2021.104803

2 M. F. El Shehry, S. Y. Abbas, A. M. Farrag, S. I. Eissa, S. A. Fouad, Y. A. Ammar, Med. Chem. Res., 2018, 27, 2287-2296. DOI:10.1007/s00044-018-2235-4

3 M. A. Salem, A. Ragab, A. A. Askar, A. El-Khalafawy, A. H. Makhlouf, Eur. J. Med. Chem., 2020, 188, 111977.

DOI:10.1016/j.ejmech.2019.111977

4 M. A. Salem, A. Ragab, A. El-Khalafawy, A. H. Makhlouf, A. A. Askar, Y. A. Ammar, Bioorg. Chem. 2020, 96, 103619. DOI:10.1016/j.bioorg.2020.103619

5 S. A. Fouad, S. A. Hessein, S. Y. Abbas, A. M. Farrag,Y. Ammar, Croat. Chem. Acta, 2018, 91, 99-107.

6 M. F. El Shehry, M. M. Ghorab, S. Y. Abbas, E. A. Fayed, S. A. Shedid, Y. A. Ammar, Eur. J. Med. Chem., 2018, 143, 14631473. DOI:10.1016/j.ejmech.2017.10.046

7 M. A. M. S. El-Sharief, S. Y. Abbas, Z. Moussa, E. W. El-Gammal, A. M. S. El-Sharief, Croat. Chem. Acta, 2018, 91, 335340. DOI:10.5562/cca3354

8 M. A. M. S. El-Sharief, S. Y. Abbas, K. A. M. El-Bayouki, E. W. El-Gammal, Eur. J. Med. Chem. 2013, 67, 263-268.

DOI:10.1016/j.ejmech.2013.06.031

9 S. Y. Abbas, M. A. M. S. El-Sharief, W. M. Basyouni, I. M. I. Fakhr, E. W. El-Gammal, Eur. J. Med. Chem., 2013, 64, 111120. DOI:10.1016/j.ejmech.2013.04.002

10 M. H. Helal, S. Y. Abbas, M. A. Salem, A. A. Farag, Y. A. Ammar, Med. Chem. Res. 2013, 22, 5598-5609.

DOI:10.1007/s00044-013-0524-5
11 G. L. Petri, V. Spano, R. Spatola, R. Holl, M. V. Raimondi, P. Barraja, A. Montalbano, Eur. J. Med. Chem., 2020, 208, 112783. DOI:10.1016/j.ejmech.2020.112783

12 V. Bhardwaj, D. Gumber, V. Abbot, S. Dhimana, P. Sharmaa, RSC Adv., 2015, 5, 15233-15266.

DOI:10.1039/C4RA15710A

13 R. P. Wurz, A. B. Charette, Org. Lett., 2005, 7, 2313-2316. DOI:10.1021/ol050442l

14 H. Lee, J. Lee, S. Lee, Y. Shin, W. Jung, J.-H. Kim, K. Park, K. Kim, H. S. Cho, S. Ro, Bioorg. Med. Chem. Lett., 2001, 11, 3069-3072. DOI:10.1016/S0960-894X(01)00624-2

15 W. W. Wilkerson, R. A. Copeland, M. Covington, J. M. Trzaskos, J. Med. Chem., 1995, 38, 3895-3901.

DOI:10.1021/jm00020a002

16 M. Koyama, N. Ohtani, F. Kai, I. Moriguchi, S. Inouye, J. Med. Chem., 1987, 30, 552-562. DOI:10.1021/jm00386a019

17 S. Cascioferro, D. Raffa, B. Maggio, M. V. Raimondi, D. Schillaci, G. Daidone, J. Med. Chem. 2015, 58, 9108-9123. DOI:10.1021/acs.jmedchem.5b00779

18 S. Cascioferro, B. Maggio, D. Raffa, M. V. Raimondi, M. G. Cusimano, D. Schillaci, B. Manachini, F. Plescia, G. Daidone, Eur. J. Med. Chem. 2016, 123, 58-68 DOI:10.1016/j.ejmech.2016.07.030

19 S. Sinha, M. Doble, S. L. Manju, Eur. J. Med. Chem. 2018, 158, 34-50. DOI:10.1016/j.ejmech.2018.08.098

20 S. O. Pember, G. L. Mejia, T. J. Price, R. J. Pasteris, Bioorg. Med. Chem. Lett. 2016, 26, 2965-2973.

DOI:10.1016/j.bmcl.2016.02.061

21 K. D. Hargrave, F. K. Hess, J. T. Oliver, J. Med. Chem. 1983, 26, 1158-1163. DOI:10.1021/jm00362a014

22 W. C. Patt, H. W. Hamilton, M. D. Taylor, M. J. Ryan, D. G. Taylor Jr., C. J. C. Connolly, A. M. Doherty, S. R. Klutchko, I. Sircar, B. A. Steinbaugh, B. L. Batley, C. A. Painchaud, S. T. Rapundalo, B. M. Michniewicz, S. C. J. Olso, J. Med. Chem. 1992, 35, 2562-2572. DOI:10.1021/jm00092a006

23 N. Ergenc, G. Capan, N.S. Gunay, S. Ozkirimli, M. Gungor, S. Ozbey, E. Kendi, Arch. Pharm. Pharm. Med. Chem. 1999, 332, 343-347. DOI:10.1002/(SICI)1521-4184(199910)332:10<34 3::AID-ARDP343>3.0.CO;2-0

24 J. C. Jaen, L. D. Wise, B. W. Caprathe, H. Tecle, S. Bergmeier, C. C. Humblet, T. G. Heffner, L. T. Meltzner, T. A. Pugsley, J. Med. Chem. 1990, 33, 311-317. DOI:10.1021/jm00163a051

25 Y. Wang, C. Wu, Q. Zhang, Y. Shan, W. Gu, S. Wang, Bioorg. Chem. 2019, 84, 468-477. DOI:10.1016/j.bioorg.2018.12.010

26 F. W. Bell, A. S. Cantrell, M. Hogberg, S. R. Jaskunas, N. G. Johansson, C. L. Jordon, M. D. Kinnick, P. Lind, J. M. Morin Jr., R. Noreen, B. Oberg, J. A. Palkowitz, C. A. Parrish, P. Pranc, C. Sahlberg, R. J. Ternansky, R. T. Vasileff, L. Vrang, S. J. West, H. Zhang, X. X. Zhou, J. Med. Chem. 1995, 38, 4929-4936.

27 S. Oncu, M. Punar, H. Erakosy, Chemotherapy 2004, 50, 98 100. DOI: $10.1159 / 000077810$

28 J. Rudolph, H. Theis, R. Hanke, R. Endermann, L. Johannsen, F. U. Geschke, J. Med. Chem., 2001, 44, 619-626. DOI: $10.1021 / \mathrm{jm} 0010623$

29 A. Srinivas, M. Sunitha, S. Shamili, Acta Chimica Slovenica, 2020, 67, 1061-1071. DOI:10.17344/acsi.2019.5752 
30 M. A. Salem, M. Helal, M. El-Gaby, Y. Ammar, M. Gouda, S. Y. Abbas, To Chem. J., 2018, 1, 114-144.

31 W. M. Basyouni, S. Y. Abbas, R. M. Dawood, K. A. M. El-Bayouki, M. K. El Awady, T. H. Abdelhafez, J. Heterocycl. Chem., 2021, 58, 1766-1774. DOI:10.1002/jhet.4307
32 F. E. Anderson, C. J. Duca, J. V. Scudi J. Am. Chem. Soc., 1951, 73, 4967-4968. DOI:10.1021/ja01154a501

33 L. Yurttaş, Y. Özkay, Z. A. Kaplancıklı, Y. Tunalı, H. Karaca, J. Enz. Inhib. Med. Chem., 2013, 28, 830-835.

DOI:10.3109/14756366.2012.688043

\section{Povzetek}

Eden izmed najboljših načinov za načrtovanje novih biocidnih spojin je sinteza hibridnih molekul, do katerih pridemo s povezavo dveh ali več bioaktivnih fragmentov v eno samo molekulsko ogrodje. Tako smo iz serije 1-metil-1H-pirol-2karbaldehid tiosemikarbazonov $\mathbf{1 a}-\mathbf{c}$ pripravili nov set pirolov, ki so vsebovali tiazolni fragment. Ciklizacija tiosemikarbazonskih derivatov 1a-c $\mathrm{z}$ etil kloroacetatom, etil 2-kloropropanoatom, kloroacetonom ali fenacil bromidom je vodila do ustreznih tiazolidin-4-onov $\mathbf{2 a - c}$, 5-metiltiazolidin-4-onov 3a-c, 4-metil-2,3-dihidrotiazolov 4a-c in 4-fenil-2,3-dihidrotiazolov $\mathbf{5 a}-\mathbf{c}$. Določili smo tudi antimikrobne aktivnosti novih tiazolskih derivatov. 